\title{
Use of $K p$ index of geomagnetic activity in the forecast of solar activity
}

\author{
G. K. Rangarajan and L. M. Barreto \\ Observatorio Nacional, São Cristovao 20921-400, Rio de Janeiro, Brazil \\ (Received January 20, 1999; Revised April 28, 1999; Accepted April 28, 1999)
}

\begin{abstract}
It is well established that geomagnetic activity in the declining phase of a solar cycle is a useful precursor of the amplitude of the solar activity in the next cycle. In the present analysis, we show that the frequency of occurrence of $K p$ index with values (0-1) representing geomagnetic calm and with values between 4 and 7 representing moderate geomagnetic disturbances in the declining phase of a solar cycle is linearly related to the sunspot maximum of the next cycle. Highest correlations are obtained when the frequencies are averaged over three years preceding the solar minimum epochs. We also show that the frequency of occurrence of consecutive 4 or 6 intervals of $K p$ with the same magnitude (0-1 or 4-5) is also equally reliable as a predictor of the ensuing solar maximum. The rate of increase of sunspot number to the solar maximum and its subsequent rate of decline to the next minimum can also be predicted reliably with these frequencies. Based on the observations for 1993-1996, we expect the coming solar maximum to be associated with an annual mean sunspot number of about 140, with a rate of ascent of 35/year and a decline rate of about 25 /year.
\end{abstract}

\section{Introduction}

Two of the most widely used indices in geophysical research are the Zurich Relative sunspot number $(R z)$ as a measure of solar activity and the index $K p$, representing geomagnetic activity in the subauroral region. Studies in solarterrestrial relationships have consistently shown the close and occasionally elusive link between the evolution of activity on the Sun and geomagnetic manifestations on the surface and in the magnetosphere. From an extensive analysis of the geomagnetic index $a a$, Legrand and Simon (1991) suggested that the solar cycle has two distinct components - dipole and toroidal-with their respective cycles being nearly out of phase. The toroidal component was shown to be strongly linked, with a 5 to 6-year delay, to the preceding dipole component. They also suggested that the solar wind flow in a coronal field is due to a dipole source. It was Ohl (1971), who first associated the recurrent geomagnetic storms to the 'ghosts' of Unipolar Magnetic Region (UMR) and showed that a close relationship existed between the intensity of the development of recurrent disturbances and the maximum of relative sunspot number $(R z M a x)$ in the following cycle indicating a link between the development of UMR towards the end of a solar cycle and of bipolar magnetic regions in the succeeding cycle. Schatten et al. (1978) later provided a dynamo-theory based physical basis for this type of association. Sofia et al. (1998) use the theory to update their estimate of the amplitude of solar activity in cycle 23 . According to them, the Sun's magnetic field has a poloidal geometry near the minimum epoch but the differential rotation in the subsequent 3-5 years wraps up the field lines into an equatorial torus. The field lines erupt through the photosphere produc-

Copy right(c) The Society of Geomagnetism and Earth, Planetary and Space Sciences (SGEPSS); The Seismological Society of Japan; The Volcanological Society of Japan; The Geodetic Society of Japan; The Japanese Society for Planetary Sciences. ing sunspots.

These and other similar findings have consequently led to the use of geomagnetic activity in the declining phase of a solar cycle as a precursor for the amplitude of the maximum solar activity in the following cycle. The better rate of success in the forecast of solar activity through geomagnetic precursors was acknowledged by Brown (1992) who found that only 10 out of 45 forecasts for solar cycle 22 (19861996) were close to the actually observed maximum $R z$ (to within 10 percent) and seven of these were precursor types. This is attributed, as mentioned above, to the fact that the Sun's future activity is determined by its polar field which are converted into toroidal field later by the differential rotation emerging as the active regions in the next cycle $(\mathrm{Li}$, 1997; Schatten and Hoyt, 1998).

Other forecast methods rely on the fact that the available data base of $R z$ is quasi-stationary and should be amenable to forecast by statistical/mathematical approaches (Kane and Trivedi, 1985; Hathaway et al., 1994; Rangarajan, 1998 and references therein).

Thomson (1993) describes a precursor technique for predicting the amplitude of the solar cycle using geomagnetic index $A p$ and claims that his method would have produced accurate predictions for all the previous cycles with a typical RMS error of only 17 in sunspot number. Rajmal Jain (1997) derives an expression linking the amplitude of the solar maximum in the succeeding cycle with the level of geomagnetic activity in the preceding descending phase. These and other methods have emphasized the role of the magnitude of the geomagnetic activity in the declining phase of a solar cycle in the forecast of the amplitude of the solar maximum.

In the present investigation, we show that the occurrence of particular levels of geomagnetic activity and their persistence over some length of time provide valuable clues not only 

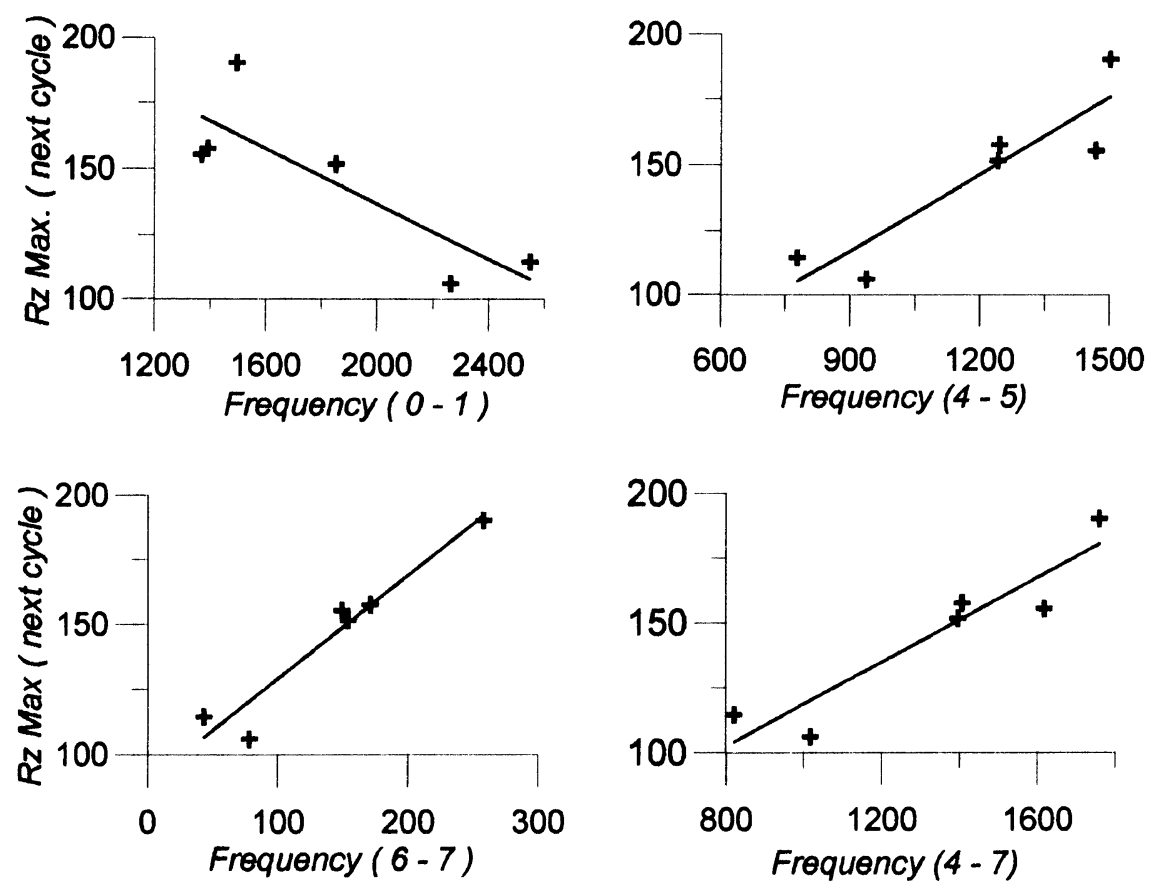

Fig. 1(a). Scatter plots of frequencies of occurrence of $K p$ index with magnitudes $0-1,4-5,6-7$ and 4-7 in the declining phase of a solar cycle against the maximum sunspot number ( $R z$ Max) of the following cycle. The straight line of best fit in the least square sense is also indicated. The frequencies are cumulatively added over two years preceding the solar minimum year (excluding the minimum).
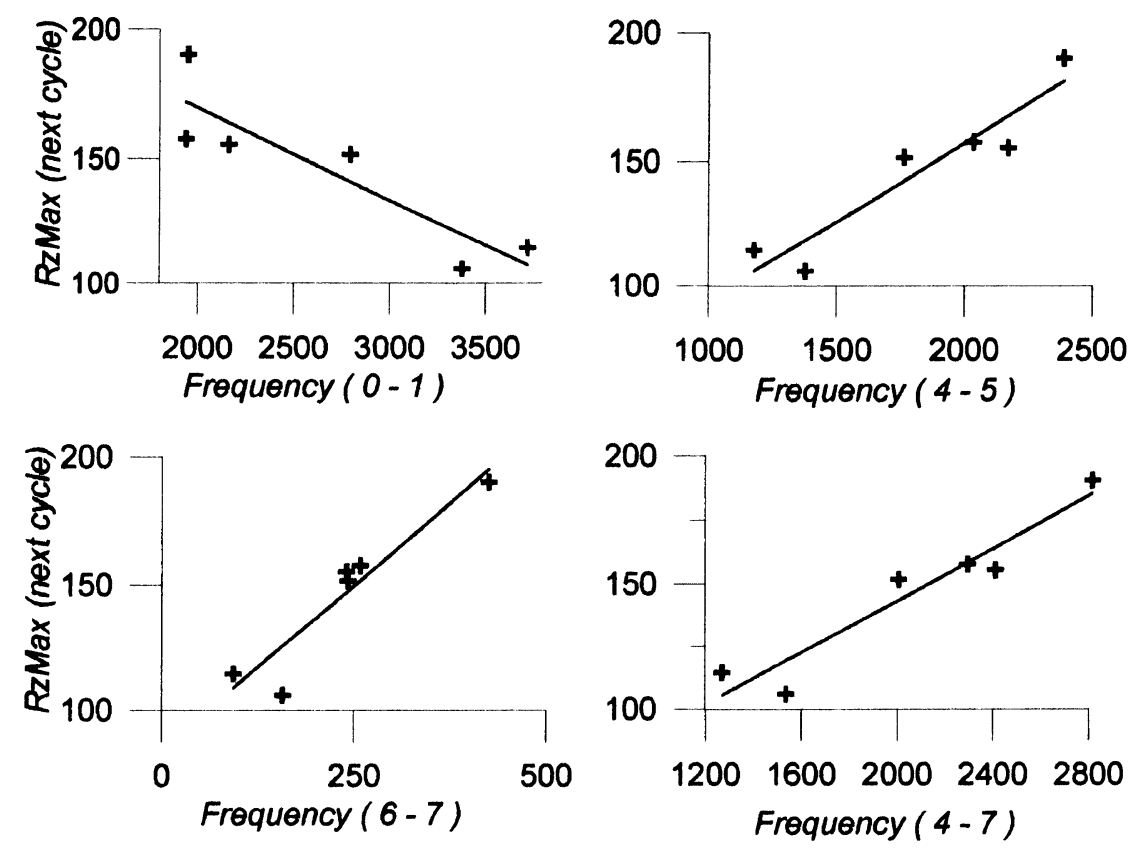

Fig. 1(b). Same as Fig. 1(a), but the cumulative sum is over three years preceding solar minimum.

to the nature of the ensuing solar maximum but also to its rate of ascent to maximum and subsequent decline to the next minimum, thereby forecasting the entire solar cycle in advance by several years.

\section{Data and Analysis}

The data base for the present work is the frequency of oc- currence, for each month, of different levels of the index $K p$ of geomagnetic activity covering the period 1932 to 1996 . The frequencies were grouped into five categories $0-1,2-3$, 4-5, 6-7 and 8-9. In addition, we also computed the cumulative numbers when the index in one of the categories occurred consecutively over 4,6 or 8 intervals (covering 12,18 or 24 hours of Universal Time). Their time variations were earlier 

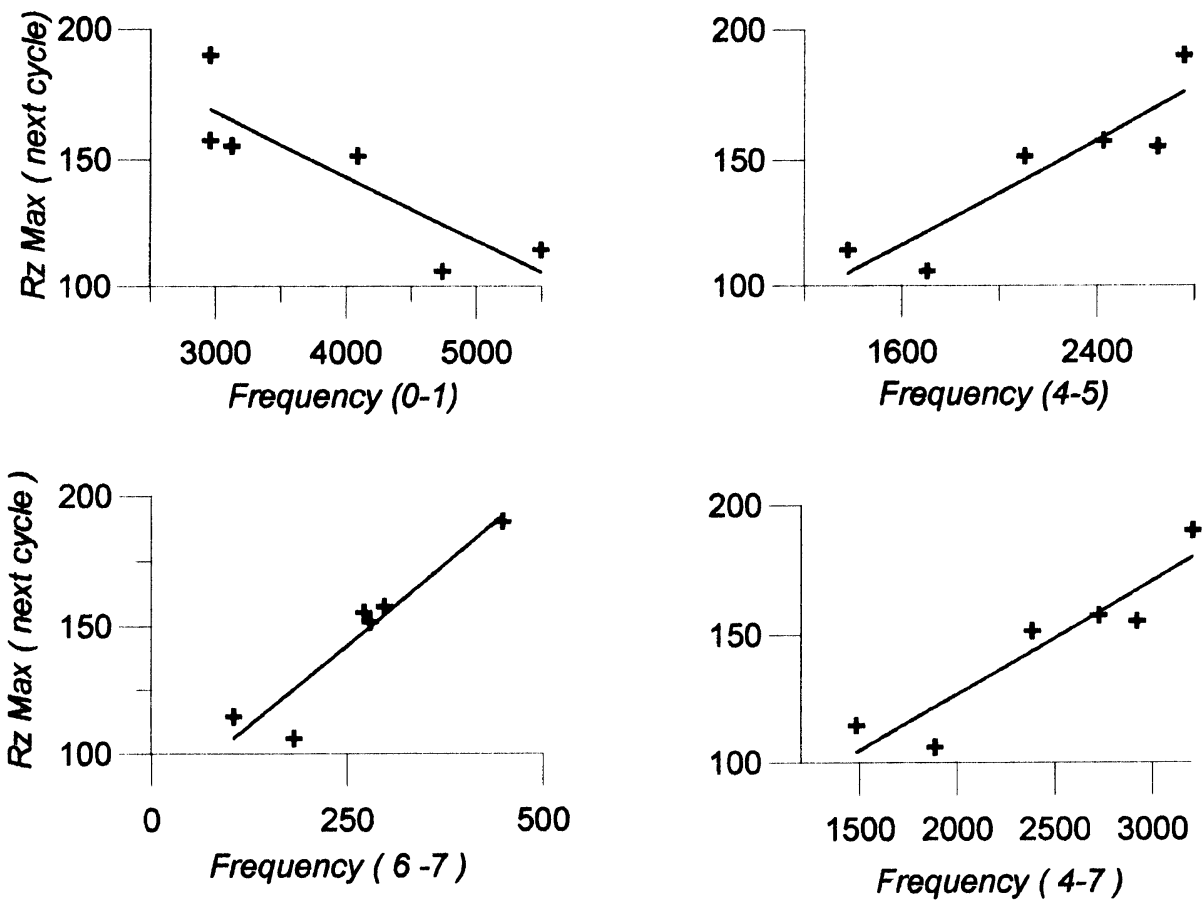

Fig. 1(c). Same as Fig. 1(a), but the cumulative sum is over four years preceding (and including) solar minimum.
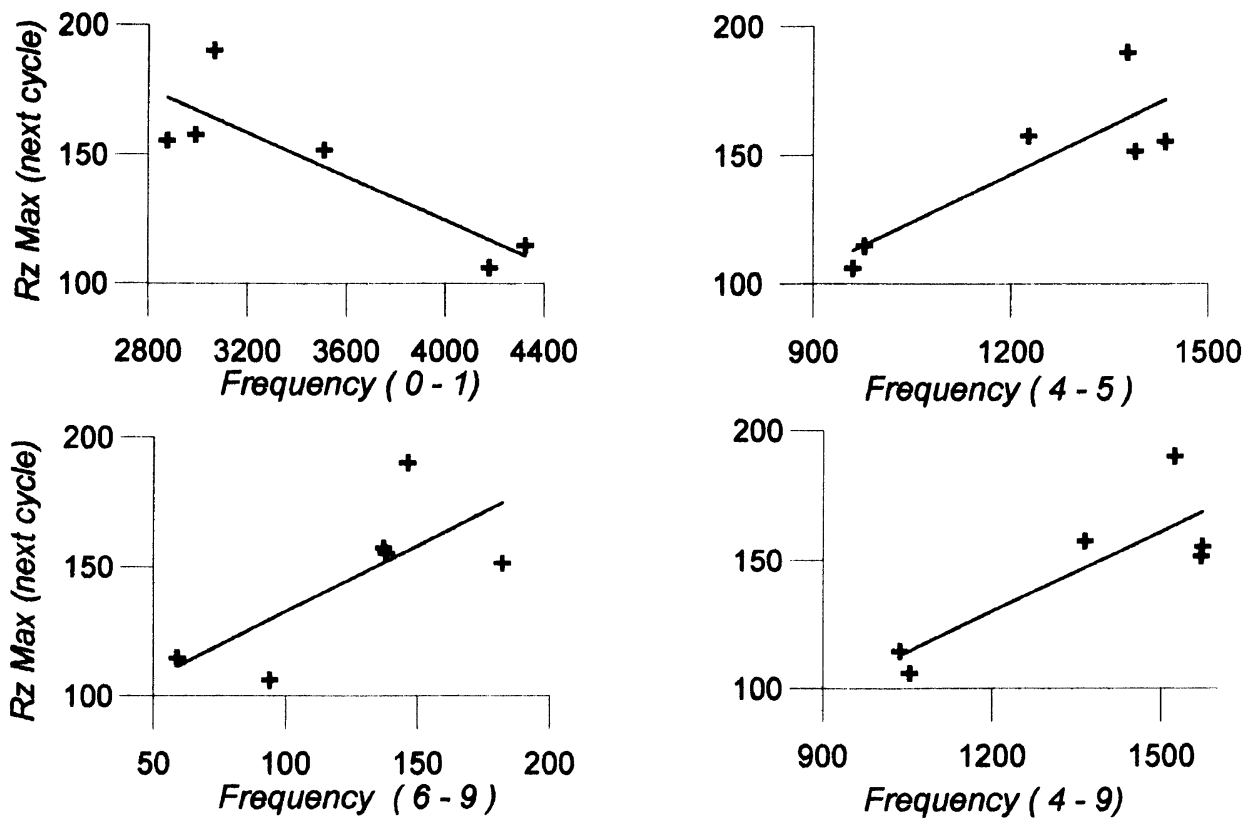

Fig. 1(d). Same as Fig. 1(a), but the cumulative sum is over three years centered on solar minimum.

analyzed exhaustively by Rangarajan and Iyemori (1997). With respect to the association between the recurrence and solar activity, most significant correlations were found to be for the group of $K p=4-5$ (4, 6 or 8 consecutive intervals) with a time lag of 6 to 7 years (see their figure 5). However, these lagged correlations were computed using the entire data of annual occurrence frequencies and $R z$, without separating them into different phases of the solar cycle.

Here, we use the list of occurrence frequencies of $K p$ for different levels of geomagnetic disturbance but restricting the same to the declining phase of each solar cycle to study its relation with the amplitude of the ensuing solar maximum. We successively use the cumulative sum over four years (including solar minimum), three years and two years (excluding the solar minimum) for the regression analysis. In addition, we also look at the possible link between these frequencies and the rate of increase of solar activity to the next maximum, and also the subsequent decline to the next 
Table 1. Correlation and Regression parameters (slope and intercept) for the scatter plots between occurrence frequencies of $K p$ with different magnitudes in the declining phase and $R z$ Max in the next cycle. Predictions, based on the regression and the occurrence frequencies during 1993-96, of $R z$ Max in solar cycle 23 are also shown.

\begin{tabular}{|c|c|c|c|c|c|c|c|c|}
\hline \multirow{2}{*}{$\begin{array}{c}\text { Years in declining } \\
\text { Phase }\end{array}$} & \multirow{2}{*}{$\begin{array}{c}K p \\
\text { Magnitude }\end{array}$} & \multirow{2}{*}{$\begin{array}{l}\text { Corr. } \\
\text { Coeff. }\end{array}$} & \multicolumn{4}{|c|}{ Regression line } & \multirow{2}{*}{$\begin{array}{c}\text { RMS } \\
\text { Departure }\end{array}$} & \multirow{2}{*}{$\begin{array}{l}\text { Predicted } \\
\text { Rz Max }\end{array}$} \\
\hline & & & Slope & Error & Inter. & Error & & \\
\hline 2 years & $0-1$ & -0.837 & -0.053 & 0.014 & 242 & 32 & 15.5 & 144 \\
\hline \multirow[t]{3}{*}{ (excl. Min.) } & $4-5$ & 0.906 & 0.098 & 0.023 & 29 & 12 & 11.8 & 156 \\
\hline & $6-7$ & 0.966 & 0.400 & 0.044 & 89 & 8 & 7.3 & 144 \\
\hline & $4-7$ & 0.934 & 0.081 & 0.013 & 37 & 10 & 10.2 & 153 \\
\hline 3 years & $0-1$ & -0.892 & -0.036 & 0.007 & 242 & 25 & 12.7 & 145 \\
\hline \multirow[t]{3}{*}{ (excl. Min.) } & $4-5$ & 0.942 & 0.062 & 0.009 & 33 & 21 & 9.6 & 148 \\
\hline & $6-7$ & 0.940 & 0.260 & 0.039 & 85 & 17 & 9.8 & 143 \\
\hline & $4-7$ & 0.955 & 0.052 & 0.007 & 40 & 9 & 8.2 & 148 \\
\hline 4 years & $0-1$ & -0.865 & -0.025 & 0.006 & 244 & 29 & 14.3 & 146 \\
\hline \multirow[t]{3}{*}{ (incl. Min.) } & $4-5$ & 0.907 & 0.052 & 0.010 & 34 & 12 & 11.8 & 142 \\
\hline & $6-7$ & 0.944 & 0.253 & 0.036 & 79 & 12 & 9.4 & 137 \\
\hline & $4-7$ & 0.927 & 0.044 & 0.007 & 38 & 10 & 10.6 & 139 \\
\hline 3 years & $0-1$ & -0.863 & 0.043 & 0.010 & 294 & 46 & 14.0 & 124 \\
\hline \multirow[t]{3}{*}{ (centred on Min.) } & $4-5$ & 0.852 & 0.125 & 0.031 & -7 & 14 & 15.0 & 108 \\
\hline & $6-7$ & 0.723 & 0.638 & 0.249 & 65 & 21 & 20.0 & 108 \\
\hline & $4-7$ & 0.846 & 0.105 & 0.027 & 4 & 14 & 14.8 & 108 \\
\hline 3 years & $0-1^{*}$ & -0.892 & -0.331 & 0.068 & 229 & 47 & 12.7 & 145 \\
\hline \multirow[t]{3}{*}{ (excl. Min.) } & $4-5^{*}$ & 0.936 & 0.669 & 0.103 & 48 & 10 & 9.8 & 154 \\
\hline & $0-1^{* *}$ & -0.898 & -0.423 & 0.104 & 209 & 17 & 12.7 & 141 \\
\hline & $4-5^{* *}$ & 0.928 & 1.179 & 0.193 & 69 & 11 & 10.6 & 158 \\
\hline
\end{tabular}

*based on four consecutive intervals with same magnitude

** based on six consecutive intervals with same magnitude

minimum so that the entire evolution of the forthcoming solar cycle can be established from the occurrence frequencies of the geomagnetic activity index in the declining phase of the preceding cycle. As the data availability is only from 1932, we extrapolated each series backwards in time over two years using an autoregressive model of the time series covering 1996-1932 period.

\section{Results and Discussion}

\subsection{Forecast of $\boldsymbol{R z}$ Max in solar cycle 23}

In Figs. 1(a), 1(b) and 1(c) we show the scatter plots and the straight lines of best fit for the occurrence frequencies with different magnitudes and the maximum sunspot number in the next cycle. $R z$ Max used in the figures correspond to the reported annual mean values of the solar maximum epochs, derived from the monthly mean values. The $K p$ occurrence frequencies correspond to cumulative sums over 2, 3 or 4 years in the declining phase. The correlation coefficients, the regression parameters with their associated standard errors, the RMS departures from linearity and the predicted values of the sunspot maximum in cycle 23 , based on the linear relationship, are given in Table 1. Frequencies of different levels of $K p$ in the declining phase of cycle 22 (1993 to 1996) are used for prediction purposes. All the forecast values are in excellent agreement except, to a limited extent, for the category $K p=4-5$ or 4-7 over two years (ref. Fig. 1(a)). All the correlation coefficients, even if they were derived from only six pairs of values, are highly significant. The errors associated with the regression coefficients are also small so that the validity of the linear relationship between $R z$ Max. (maximum sunspot number of the next cycle) and any one of the four frequency parameters, appear to be real and striking. 

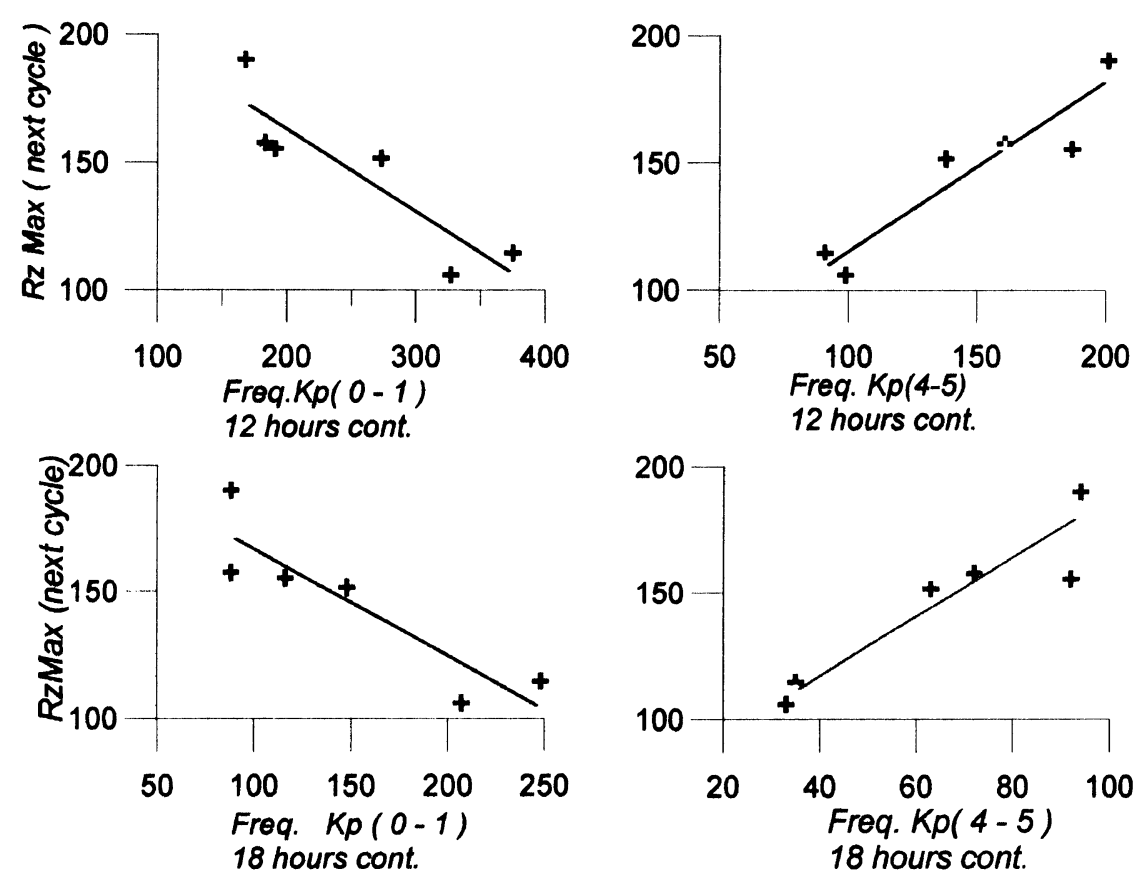

Fig. 2. Scatter plots of $R z$ Max against frequencies of occurrence of consecutive intervals of same magnitude of $K p$ index (0-1 or 4-5 over four or six consecutive intervals) and the straight lines of best fit to the plot.

The RMS departures from linearity is only about 10 . It may also be noted that for solar cycles 18, 21 and $22 \mathrm{Rz}$ Max values were nearly equal (between 152 and 158) but they fit into the linear trend nicely in many of the scatter plots between the extreme values of 190 and 106 of cycles 19 and 20 respectively.

The best results are seen when frequencies are reckoned over three years preceding the solar minimum. These figures reconfirm the strong link between the recurrent magnetic disturbance in the declining phase and the ensuing solar maximum. Interestingly, the occurrence of very quiet geomagnetic conditions (with $K p=0$ or 1) also appear to be an equally valuable candidate for forecast purposes!

To see whether the strong linear relationship between the frequencies of $K p$ and $R z M a x$ is confined only to the declining phase, we examine the relation between the occurrence frequencies, now confined to three years centered on the solar minimum epoch. The corresponding scatter plots and the line of best fit are shown in Fig. 1(d). Not surprisingly, the correlations are now much weaker, RMS departures and the errors associated with the regression parameters are relatively higher. This figure conclusively shows that the established relation between different levels of geomagnetic activity in the descending phase of the solar cycle and $R z$ Max in the next cycle is not due to chance and once the solar minimum is reached, there is a possible breakdown in the observed features.

We next consider whether persistence in the occurrence of a given level of geomagnetic activity could be an indicator of the magnitude of solar activity to follow. For this purpose, we computed the frequency of occurrence of $K p$ with values 0-1 or 4-5 over four, six or eight consecutive intervals (covering 12, 18 or 24 hours of Universal Time) for each year in the declining phase of each solar cycle. The numbers for 8 consecutive occurrences turned out to be quite small and, hence, not utilized. The scatter plots of occurrence frequencies of consecutive quiet or moderately disturbed intervals and $R z$ Max are shown in Fig. 2. The regression parameters and the predicted values of $R z$ Max for cycle 23 are also included in Table 1 . We notice that when moderately disturbed conditions, represented by $K p=4-5$, persist for 12 to 18 hours, their cumulative frequencies are excellent indicators of $R z$ Max. The RMS departures are only 10 for a predicted maximum of about 155 . Consecutive occurrences of quiet intervals $(K p=0-1)$ appear to be only slightly less effective as precursors of $R z$ Max, in this case.

Let us examine how the predicted value (of $145+/-10$ ) for solar cycle 23 compares with others. Nevanlinna and Kataja (1993) obtained a linear relation

$$
R(M)=14.6+8.1 a a
$$

where $R(M)$ is the smoothed maximum sunspot number and $a a$ is the minimum annual mean value of $a a$. Using a value of 16.2 for the annual mean value of $a a$ for 1997, we get the expected $R(M)$ in cycle 23 to be 146 . Schatten and Myers (1996), using two properties of solar activity prediction techniques - that solar magnetism oscillates between poloidal and toroidal components; and that there is a degree of "magnetic persistence" dynamos-anticipate a $R z$ maximum of $138+/-30$. They find that, contrary to the general observation that odd cycles have greater amplitude of sunspot variation, cycle 23 may be weaker than cycle 22. Sofia et al. (1998) give an update of their prediction for cycle 23 with a value of $143+/-30$ and suggest that the value is comparable to an alternative prediction of 138, based on the magnitude of the IMF. Recently Rangarajan (1998) studied the variability 

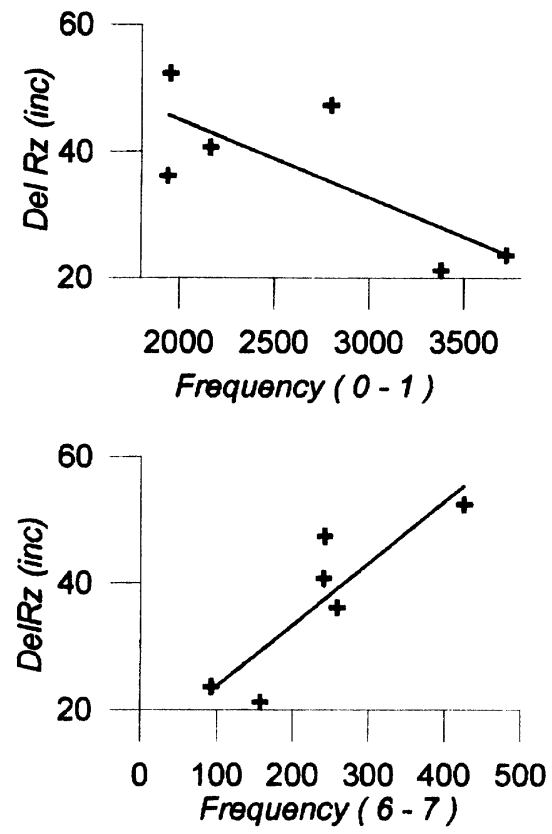
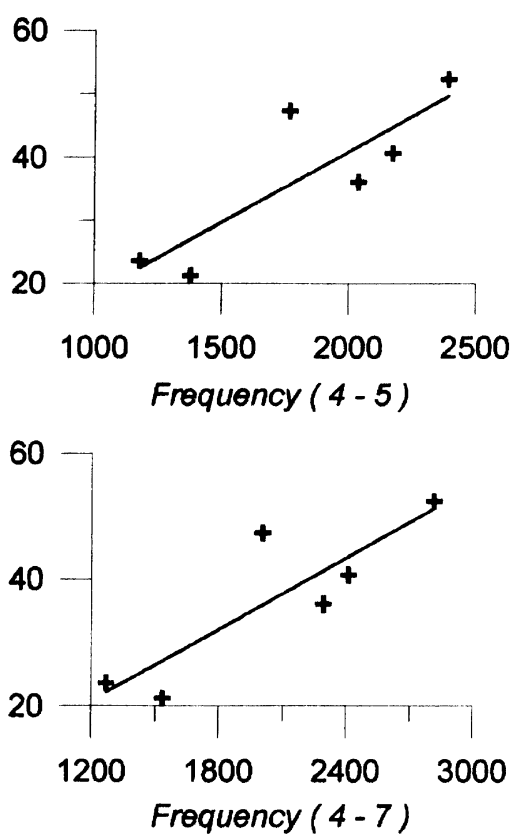

Fig. 3(a). Scatter plots of rate of increase of $R z$ from solar minimum to maximum epochs against cumulative frequencies of $K p$ index with magnitudes $0-1,4-5,6-7$ and $4-7$. The sum is taken over three years preceding solar minimum.
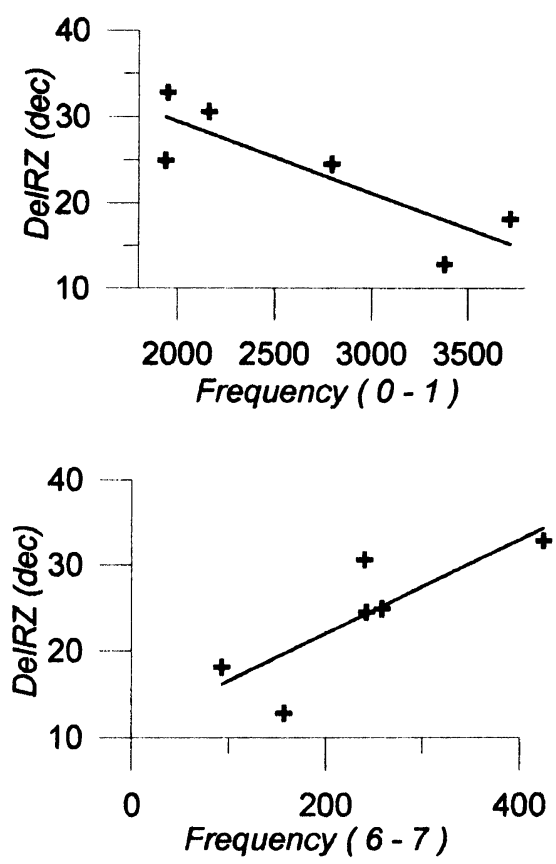
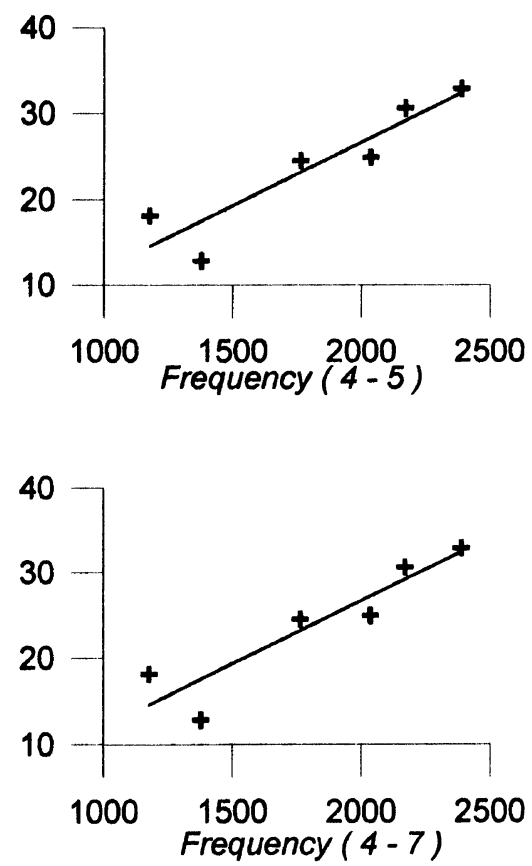

Fig. 3(b). Same as Fig. 3(a) but the rate of decrease of $R z$ from a year after the maximum to the next minimum epoch is taken.

of sunspot numbers over the entire period of availability of $R z$ data (1750 to present) through a data adaptive filtering technique, called Singular Spectrum Analysis (see Vautard et al., 1992 for details) and estimated that the ensuing solar maximum will have an associated $R z$ Max of about 130 .

Decomposing the $A p$ index into two parts-one linearly dependent on sunspot number and the other, the residual part- , Li (1997) forecast a value of $149+/-20$ for cycle
23 maximum. Joselyn et al. (1997) predict that cycle 23 will be associated with a smoothed sunspot maximum of 160 , nearly on par with the previous cycle. They also provide associated error intervals for their prediction and their lower limit is practically the same as we have suggested from the present analysis. All these forecasts may be considered to be in fair agreement with each other and close to the values forecast by the present approach. 
Table 2. Correlation and regression parameters (slope and intercept) for the scatter plots between occurrence of $K p$ with different magnitudes in the declining phase and the following rate of increase/decrease of $R z$ from solar minimum to maximum (year after solar maximum to minimum) as also for the scatter plots between $R z$ Max and the occurrence frequencies of different magnitudes of $K p$ centered on 3 or 5 years over the same epoch.

\begin{tabular}{|c|c|c|c|c|c|c|c|c|}
\hline \multirow{2}{*}{$\begin{array}{c}\text { Years in solar cycle } \\
\text { Phase }\end{array}$} & \multirow{2}{*}{$\begin{array}{c}K p \\
\text { Magnitude }\end{array}$} & \multirow{2}{*}{$\begin{array}{l}\text { Corr. } \\
\text { Coeff. }\end{array}$} & \multicolumn{4}{|c|}{ Regression line } & \multirow{2}{*}{$\begin{array}{c}\text { RMS } \\
\text { Departure }\end{array}$} & \multirow{2}{*}{$\begin{array}{c}\text { Predicted } \\
\text { Rate }\end{array}$} \\
\hline & & & Slope & Error & Inter. & Error & & \\
\hline 3 years & $0-1$ & -0.756 & -0.012 & 0.005 & 70 & 15 & 4.3 & 37.2 \\
\hline (excl. min.) & $4-5$ & 0.844 & 0.022 & 0.007 & -4 & 13 & 3.9 & 36.8 \\
\hline Rate of inc. & $6-7$ & 0.870 & 0.097 & 0.023 & 14 & 7 & 3.7 & 35.6 \\
\hline of $R z$ & $4-7$ & 0.861 & 0.019 & 0.005 & -2 & 5 & 3.8 & 37.8 \\
\hline Rate of dec. & $0-1$ & -0.849 & -0.008 & 0.002 & 46 & 7 & 3.0 & 24.3 \\
\hline \multirow[t]{3}{*}{ of $R z$} & $4-5$ & 0.919 & 0.015 & 0.003 & -3 & 6 & 3.6 & 24.8 \\
\hline & $6-7$ & 0.816 & 0.055 & 0.016 & 11 & 5 & 3.1 & 23.3 \\
\hline & $4-7$ & 0.912 & 0.012 & 0.002 & -1 & 6 & 2.6 & 23.9 \\
\hline 5 years & $0-1$ & -0.940 & -0.024 & 0.004 & 263 & 22 & 24 & - \\
\hline \multirow[t]{3}{*}{ (centered on Max) } & $4-5$ & 0.957 & 0.062 & 0.008 & -3 & 23 & 20 & - \\
\hline & $6-9$ & 0.953 & 0.048 & 0.006 & 9 & 9 & 21 & - \\
\hline & $4-9$ & 0.906 & 0.203 & 0.039 & 54 & 12 & 29 & - \\
\hline 3 years & $0-1$ & -0.945 & -0.035 & 0.005 & 246 & 18 & 23 & - \\
\hline \multirow[t]{3}{*}{ (centered on Max) } & $4-5$ & 0.939 & 0.092 & 0.014 & 9 & 10 & 24 & - \\
\hline & $6-9$ & 0.834 & 0.249 & 0.069 & 74 & 15 & 38 & - \\
\hline & $4-9$ & 0.919 & 0.068 & 0.012 & 25 & 11 & 27 & - \\
\hline
\end{tabular}

On the other hand, Wilson (1992) insists that the Rz Max for cycle 23 should be greater than 176 and gave an estimate of $214+/-37$. He based his forecast on two specific features of solar activity: (i) that there is a long term increase of the maximum amplitude with time and (ii) in last six even/odd cycle pairs, odd cycles have, on the average, about 40 units higher values of Rz Max. According to him, cycle 23 should be potentially one of the greatest cycles of the modern era. Wilson et al. (1998a) later predicted a probable value of 168 which was further revised to a lower figure of 144 (Wilson et al., 1998b) with the availability of the most recent observations of the evolution of solar activity after the minimum in May/August 1996. Thomson (1993) showed that the number of geomagnetic disturbances in the current solar cycle (indicated by $A p>=25$ ) can be utilized in an expression relating this to the amplitude of $R z$ in the current and next cycles. Using either of his suggested equations and 637 as the number of days with $A p>=25$ in solar cycle 22, we get an estimate of 178 for the $R z$ Max in cycle 23. Rajmal Jain (1997), adopting the average value of $a$ a index for the sunspot minimum year and preceding four years of a given cycle as a precursor to the $R z$ Max of the following cycle, predicts a value of 166 for cycle 23. Basing his estimation of the solar maximum amplitude on the statistics of extremes, Silbergleit (1998) suggests that it may be expected to be $173+/-14$.
We thus find that the forecasts of the Rz Max for the coming cycle broadly lie in two bands: one with value of about 140 and the other higher at about 170 to 180 . Interestingly, the RMS departure of the predictions arrived at by both Thomson (1993) and by the present analysis is only about 10, even if the predictions themselves differ significantly. Coming years will certify which turns out to be more near reality! The main advantage of the present method is the fact that we have several possible alternatives to arrive at an estimate of $R z$ Max, all based only on the occurrence of different levels of Kp index. This index is derived quite objectively, has been shown to be a useful proxy for many solar wind and IMF parameters and with digital alternatives to the conventional $K$-index derivation, will be available without much delay. If the predictions prove reliable in the coming years, one can reconstruct the frequencies of occurrence of different levels of geomagnetic activity in the past solar cycles before 1932 , as reliable sunspot data dates back to 1750 !

\subsection{Forecast of the rate of increase and decrease of $R z$ in a solar cycle}

It is easily noticeable that when the maximum $R z$ in a solar cycle is larger in amplitude, the rate of ascent from minimum is faster. Nordemann (1992) examined the possibility of fitting exponential functions separately for the ascending and declining phases of each solar cycle. He found that the 

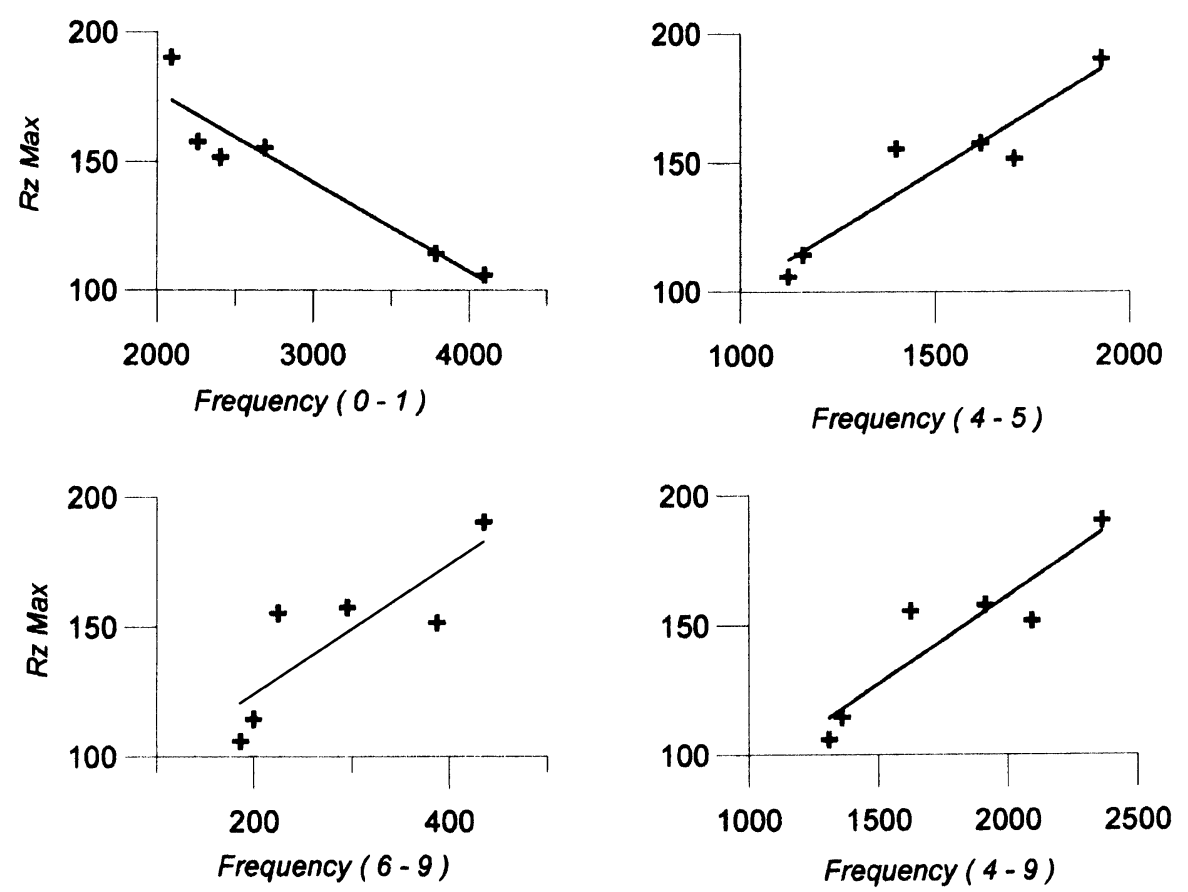

Fig. 4(a). Scatter plots of $R z$ Max against cumulative frequencies of $K p$ index with magnitudes 0-1, 4-5, 6-9 and 4-9, where the sum is taken over three years centered on the epoch of solar maximum.
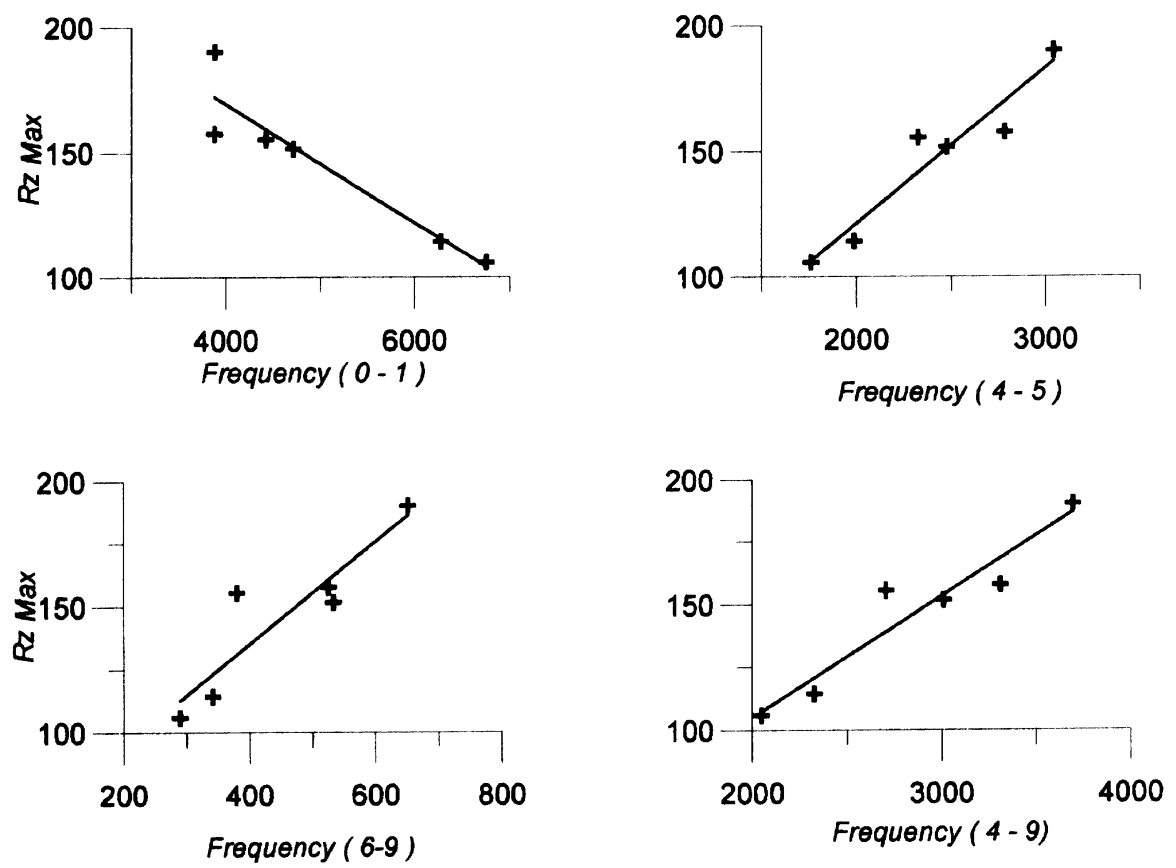

Fig. 4(b). Same as Fig. 4(a) but the cumulative sum is taken over five years centered on the epoch of solar maximum.

exponent showed a narrow distribution of values for the declining phase and a broader distribution for the rising phase. This suggests that the decline of $R z$, in any of the 27 solar cycles that he considered, has a fairly well-ordered behavior in contrast to the ascent to maximum. Hathaway et al. (1994) attempted description of the temporal behavior of the sunspot series with four parameters: starting time, ampli- tude, rise time and asymmetry. The asymmetry between rise to maximum and fall to minimum was found to be almost invariant from cycle to cycle and a close relationship was seen between rise time and amplitude of the cycle. Since the maximum value of $R z$ in a cycle is shown to be associated with the geomagnetic activity in the preceding declining phase, it is reasonable to expect a functional relationship between the 
rate of increase of $R z$ from minimum to maximum epoch and the geomagnetic activity index in the previous cycle. Such a linear relationship was shown to exist between the minimum value of $A a$ index and the rate of increase in $R z$ by Rangarajan (1977).

Based on these considerations, we examine the relation between the occurrence frequencies of $K p$ index with different magnitudes in the declining phase of any solar cycle with the rate of increase of $R z$ in the next cycle. As the best correlations were earlier obtained when the data was confined to three years preceding the solar minimum (excluding the solar minimum), we present results only for this subset. The rate of increase of $R z$ is reckoned as the average of the difference between the maximum and minimum $R z$ in the following cycle. For the rate of decrease, we take one year after the maximum to the next solar minimum, because of the persistence of maximum values, on occasions, over two or three years. In Figs. 3(a) and 3(b), we show the scatter plots of the occurrence frequencies of different levels of geomagnetic activity and the rates of increase (decrease) of $R z$. The corresponding regression parameters are listed in Table 2, together with the predicted values.

These figures reveal that the dependence of the rate of change of $R z$ on the geomagnetic activity is not quite as linear as was found for the amplitude in the earlier section. However, taking into consideration the errors involved, a reasonable forecast of the rate of ascent to be about 35 /year for cycle 23 . If the cycle maximum is expected in $2000 \mathrm{AD}$, as forecast by Schatten and Myers (1996), the forecast maximum will be about 140 , consistent with the figures arrived at earlier, and once again much less than the forecast of Wilson (1992) or Thomson (1993). However, if the revised forecast of Wilson et al. (1998b) are valid, then the rate of increase of $R z$ from minimum to the expected maximum in mid-2000 will be practically the same, as reported by us. Our forecast for the rate of decrease of $R z$ from its maximum value in cycle 23 is $24 /$ year. This implies that the declining phase is expected to last about 5 years before the minimum solar activity is reached.

\subsection{Relation between occurrence frequencies of $K p$ and $R \boldsymbol{z}$ in the maximum phase of the solar cycle}

Feynman (1982) showed that geomagnetic activity index can be split into a part linearly dependent on solar activity and a second one independent of sunspot number. Legrand and Simon (1991) studied the topology of the solar coronal field and showed that geomagnetic activity, as represented by SSC storms distributed at random, is linked to sunspot activity whereas recurrent storms are associated with corotating high speed solar wind streams. In his approach to the prediction of the amplitude of a solar cycle, Thomson (1993) also considered that the contribution to geomagnetic disturbances comes from two distinct sources, one of which is linked to the solar maximum epoch. It is, therefore, reasonable to expect that the occurrence frequencies of different levels of geomagnetic activity during the maximum phase of a solar cycle can be directly related to the maximum sunspot number in the same epoch.

We examine this aspect next by carrying out a regression analysis of the occurrence frequencies of $K p$ index and annual mean $R z$ centered on solar maximum epochs. Figures 4(a) and 4(b) show that near-linear relation exists between occurrence frequencies of different levels of the $K p$ index, cumulatively added over either three years or five years centered on the solar maximum and $R z$ Max in the same epoch. The correlation coefficients are very significant and the RMS departures are small. Interestingly, once again, we find that occurrence of quiet intervals with magnitude of $K p=0-1$, is an excellent proxy for the $R z$ max. This linear relationship cannot be a prognostic tool for any estimate of the solar cycle, as it involves frequencies to be computed after the solar maximum is reached. However, as mentioned earlier, with the sunspot series available from 1750, we can get a reliable estimates of the occurrences of quiet and disturbed intervals in the past when no geomagnetic measurements were made.

\section{Conclusion}

While it has been fairly well established that the magnitude of geomagnetic activity in the declining phase of a solar cycle is a useful precursor for the amplitude of the sunspot number in the following cycle, we have shown, for the first time, that occurrence frequencies of different levels of geomagnetic activity including geomagnetic calm intervals, can be very useful forecast tools for the amplitude of $R z$ Max. In addition, they seem to provide adequate advance information on the evolution of the entire solar cycle, indicative of the rate of ascent to the solar maximum and the subsequent decline to minimum. Based on the occurrence frequencies of different magnitudes of $K p$ index between 1993 and 1996, in the declining phase of cycle 23 , we estimate that the ensuing solar cycle will be associated with a maximum sunspot number of about 140, a rate of increase to maximum of about $35 /$ year and a subsequent decline to minimum at the rate of about 24/year. As all the established linear relationships are statistically quite significant with small associated RMS departures, we also suggest that the sunspot numbers in the past era could be used in getting reliable estimates of the state of geomagnetic activity in the declining and maximum phases of solar activity, during intervals when geomagnetic measurements were not available.

Acknowledgments. GKR is grateful to CNPq Brazil for the offer of a fellowship to work as a Visiting Professor at Observatorio Nacional. The authors are thankful to the World Data Center for Geomagnetism and Space Magnetism, Kyoto University which provided access to the data files of $K p$ and $R z$.

The Editor thanks Jo Ann Joselyn and T. Watanabe for their assistance in evaluating this paper.

\section{References}

Brown, G. M., The peak of solar cycle 22: prediction in retrospect, Ann. Geophys., 10, 453-461, 1992.

Feynman, J., Geomagnetic and solar wind cycles, 1900-1975, J. Geophys. Res., 87, 6153-6162, 1982.

Hathaway, D. H., R. M. Wilson, and E. J. Reichmann, The shape of the sunspot cycle, Sol. Phys., 151, 177-190, 1994.

Joselyn, J. A., J. B. Anderson, H. Coffey, K. Harvey, D. Hathaway, G. Hackman, E. Hildner, W. Mende, K. Schatten, R. Thompson, A. W. P. Thompson, and O. R. White, Panel achieves consensus prediction of solar cycle 23, EOS Trans. AGU, 78(20), 205, 211, 212, 1997.

Kane, R. P. and N. B. Trivedi, Periodicities in sunspot numbers, J. Geomag. Geoelectr., 37, 1071-1085, 1985.

Legrand, J. P. and P. A. Simon, A two-component solar cycle, Sol. Phys., 131, 187-209, 1991.

Li, Y., Predictions of the features for sunspot cycle 23, Sol. Phys., 170, 
437-445, 1997.

Nevanlinna, H. and E. Kataja, An extension of the geomagnetic activity index series aa for two solar cycles (1844-1868), Geophys. Res. Lett., 20, 2703-2706, 1993.

Nordemann, D. J. R., Sunspot number time series: exponential fitting and solar behavior, Sol. Phys., 141, 199-202, 1992.

Ohl, A. I., Physics of the 11 year variation of geomagnetic disturbances, Geomagn. Aeron., 11, 549-550, 1971.

Rajmal Jain, Prediction of the amplitude of sunspot cycle 23, Sol. Phys., 176, 431-437, 1997.

Rangarajan, G. K., Geomagnetic activity, sunspot number and its rate of increase, Proc. Indian Acad. Sci., 85(A), 71-74, 1977.

Rangarajan, G. K., Sunspot variability and an attempt to predict solar cycle 23 by adaptive filtering, Earth Planets Space, 50, 91-100, 1998.

Rangarajan, G. K. and T. Iyemori, Time variations of geomagnetic indices Kp and Ap: an update, Ann. Geophysicae, 15, 1271-1290, 1997.

Schatten, K. H. and D. Hoyt, Solar cycle 23 forecast update, Geophys. Res. Lett., 25, 599-601, 1998.

Schatten, K. H. and D. J. Myers, Solar activity forecast for solar cycle 23, Geophys. Res. Lett., 23, 605-608, 1996.

Schatten, K. H., P. H. Scherrer, L. Svalgaard, and J. M. Wilcox, Using dynamo theory to predict the sunspot number during solar cycle 21, Geophys. Res. Lett., 5, 411-414, 1978.

Silbergleit, V. M., On the statistics of maximum sunspot numbers, J. Atmos. Sol. Terr. Phys., 60, 1707-1710, 1998.

Sofia, S., P. Fox, and K. H. Schatten, Forecast update for activity cycle 23 from a dynamo-based method, Geophys. Res. Lett., 25, 4149-4152, 1998

Thomson, R. J., A technique for predicting the amplitude of the solar cycle, Sol. Phys., 148, 383-388, 1993.

Vautard, R., P. Yiou, and M. Ghil, Singular spectrum analysis: a toolkit for short, noisy chaotic signal, Physica, D58, 95-126, 1992.

Wilson, R. M., An early estimate for the size of cycle 23, Sol. Phys., 140, 181-193, 1992.

Wilson, R. M., D. H. Hathaway, and E. J. Reichmann, An estimate for the size of cycle based on near-minimum conditions, J. Geophys. Res., 103, 6595-6603, 1998a.

Wilson, R. M., D. H. Hathaway, and E. J. Reichmann, Estimating the size and timing of maximum amplitude for cycle 23 from its early cycle behavior, J. Geophys. Res., 103, 17411-17418, 1998b.

G. K. Rangarajan (e-mail: ranga@dge1.on.br) and L. M. Barreto 